\title{
THE GROUP OF ISOMETRIES ON HARDY SPACES OF THE $n$-BALL AND THE POLYDISC
}

\author{
by EARL BERKSON $\dagger$ and HORACIO PORTA
}

(Received 7 June, 1979)

1. Introduction. Let $\mathbf{C}$ be the complex plane, and $U$ the disc $|z|<1$ in $\mathbf{C}$. $\mathbf{C}^{n}$ denotes complex $n$-dimensional Euclidean space, $<,>$ the inner product, and $|\cdot|$ the Euclidean norm in $\mathbf{C}^{n}$. $B_{n}$ will be the open unit ball $\left\{z \in \mathbf{C}^{n}:|z|<1\right\}$, and $U^{n}$ will be the unit polydisc in $\mathbf{C}^{n}$. For $1 \leq p<\infty, p \neq 2, G^{p}\left(B_{n}\right)$ (resp., $G^{p}\left(U^{n}\right)$ ) will denote the group of all isometries of $H^{\mathrm{p}}\left(B_{n}\right)$ (resp., $H^{\mathrm{p}}\left(U^{n}\right)$ ) onto itself, where $H^{p}\left(B_{n}\right)$ and $H^{\mathrm{p}}\left(U^{n}\right)$ are the usual Hardy spaces.

In this paper we study some connectedness questions for $G^{p}\left(B_{n}\right)$ and $G^{p}\left(U^{n}\right)$ in the uniform and strong operator topologies (\$2). It turns out that for all $n, G^{p}\left(B_{n}\right)$ is connected in the strong operator topology, but for $n>1, G^{p}\left(U^{n}\right)$ is not. For $n \geq 1$, either group, in the uniform operator topology, is close to being totally disconnected-more precisely, the component of any $T$ consists of all products $\lambda T$, where $\lambda$ is a unimodular complex number. A pleasant byproduct of these considerations is the fact that, in the space of composition operators on $H^{p}(U)$, the identity operator is an isolated point with respect to the uniform operator topology (Corollary (2.10) below). In $\$ 3$ we show that $G^{p}\left(B_{n}\right)$ and $G^{p}\left(U^{n}\right)$ have only the trivial invariant subspaces. This extends the result for $n=1$ in $[\mathbf{1}]$.

In what follows, we denote by $K$ the unit circle $\{z \in \mathbf{C}:|z|=1\}$. The distinguished boundary of $U^{n}$ is thus the torus $K^{n}$. Normalized Lebesgue measure on $K^{n}$ will be written $m_{n}$. The boundary $S$ of $B_{n}$ has a unique rotation-invariant Borel probability measure, denoted by $\sigma$ (the suppression of any mention of $n$ in the symbols $S$ and $\sigma$ should not cause any confusion). We write Aut $\left(B_{n}\right)$ for the set of all one-to-one holomorphic maps of $B_{n}$ onto $B_{n}$.

For a general treatment of isometries of Hardy spaces, including a discussion of the history of the following two propositions, we refer the reader to [3].

(1.1) Proposition (Forelli, Rudin). Suppose $0<p<\infty, p \neq 2, n$ is a positive integer, and $T$ is a linear isometry of $H^{p}\left(B_{n}\right)$ onto $H^{p}\left(B_{n}\right)$. Then there are $\Phi \in \operatorname{Aut}\left(B_{n}\right)$ and $\lambda \in K$ such that

$$
(T f)(z)=\lambda\left\{\left(1-|a|^{2}\right) /(1-\langle z, a\rangle)^{2}\right\}^{n / p} f(\Phi(z))
$$

for all $f \in H^{p}\left(B_{n}\right), z \in B_{n}$, where $a \in B_{n}, \Phi(a)=0$. Moreover, if $n, \Phi, a$, and $\lambda$ are as above, then (1.2) defines an isometry of $H^{p}\left(B_{n}\right)$ onto $H^{\mathrm{p}}\left(B_{n}\right)$ for $0<p<\infty$.

† The work of this author was supported by a National Science Foundation grant.

Glasgow Math J. 21 (1980) 199-204. 
(1.3). Propostrion (R. B. Schneider). Suppose $0<p<\infty, p \neq 2, n$ is a positive integer, and $T$ is a linear isometry of $H^{p}\left(U^{n}\right)$ onto $H^{p}\left(U^{n}\right)$. Then there are Möbius transformations of $U, \Phi_{i}, j=1,2, \ldots, n$, a permutation $\alpha$ of $\{1,2, \ldots, n\}$, and $\lambda \in K$ such that

$$
(T f)(z)=\lambda\left[\prod_{j=1}^{n}\left(\Phi_{j}^{\prime}\right)^{1 / p}\left(z_{\alpha(j)}\right)\right] f\left(\Phi_{1}\left(z_{\alpha(1)}\right), \ldots, \Phi_{n}\left(z_{\alpha(n)}\right)\right)
$$

for all $f \in H^{p}\left(U^{n}\right), z=\left(z_{1}, z_{2}, \ldots, z_{n}\right) \in U^{n}$. On the other hand, if $n, \Phi_{1}, \ldots, \Phi_{n}, \alpha$, and $\lambda$ are as above, then (2.4) defines a linear isometry of $H^{p}\left(U^{n}\right)$ onto $H^{p}\left(U^{n}\right)$ for $0<p<\infty$.

The authors are indebted to $\mathrm{R}$. Kaufman for valuable conversations.

2. Connectedness in the Strong and Uniform Operator Topologies. For $p=2$, the operators of the form (1.2) (resp., (1.4)) obviously form a subgroup of the unitary group of $H^{2}\left(B_{n}\right)$ (resp., $H^{2}\left(U^{n}\right)$, and we denote this subgroup by $G^{2}\left(B_{n}\right)$ (resp., $G^{2}\left(U^{n}\right)$ ). For $T \in G^{p}\left(B_{n}\right), 1 \leq p<\infty$, the expression in braces on the right of $(1.2)$ has the analytic logarithm $2 \log \left(\sqrt{1-|a|^{2}} /(1-\langle z, a\rangle)\right)$, where "Log" is the principal branch of the logarithm. We shall standardize the $n / p$-power of this expression by using this analytic logarithm. It follows that the representation of $T$ in (1.2) is unique. For $T \in G^{p}\left(U^{n}\right)$, $1 \leq p<\infty$, the $n$-tuple $\left(\Phi_{1}, \Phi_{2}, \ldots, \Phi_{n}\right)$ and the permutation $\alpha$ on the right of (1.4) are uniquely determined by $T$. It is easy to see that the correspondence $T \mapsto \alpha$ is a homomorphism of $G^{p}\left(U^{n}\right)$ onto the symmetric group on $\{1,2, \ldots, n\}$.

(2.1) TheORem. For $1 \leq p<\infty, G^{\mathrm{p}}\left(B_{n}\right)$ is connected in the strong operator topology.

Proof. Let $T \in G^{p}\left(B_{n}\right)$, and consider the expression on the right of (1.2). Write $\lambda=e^{i \theta}$, for some real number $\theta$, and choose coordinates so that $a=(r, 0, \ldots, 0), r \geq 0$. Put $s=\sqrt{1-r^{2}}$. Then there is a Hermitian operator $A$ on $\mathbf{C}^{n}$ such that for all $z \in B_{n}$

$$
\Phi(z)=\left(1-r z_{1}\right)^{-1} e^{i A}\left(z_{1}-r, s z_{2}, \ldots, s z_{n}\right) .
$$

For $0 \leq t \leq 1$, let $\Phi_{t} \in \operatorname{Aut}\left(B_{n}\right)$ be obtained by replacing $r$ and $A$ in (2.2) by $t r$ and $t A$ (with the corresponding replacement in $s$ ). Let $T_{t}$ be obtained by replacing $\lambda$, $a$, and $\Phi$ in (1.2) by $e^{i t \theta}, t a$, and $\Phi_{t}$, respectively. Clearly $T_{1}=T$, and $T_{0}$ is the identity operator. It is easy to see that $T_{t}$ is a continuous function of $t$ in the strong operator topology, by showing that for each polynomial $P$, the boundary function of $T_{t} P$ is a continuous function of $t$ into $L^{p}(\sigma)$.

(2.3) THEOREM. For $1 \leq p<\infty$, let $\gamma_{p}=\left[\int_{K}|z-1|^{p} d m_{1}(z)\right]^{1 / p}$. For $n$ a positive integer, let $Z_{k}(z)=z_{k},\left(z \in \mathbf{C}^{n}, k=1,2, \ldots, n\right)$. If $T \in G^{p}\left(U^{n}\right)$ and $\|T(1)-1\|_{p}<\gamma_{p} / 2,\left\|T Z_{k}-Z_{k}\right\|_{p}<$ $\gamma_{\mathrm{p}} / 2,(k=1,2, \ldots, n)$ then the permutation $\alpha$ on the right of (1.4) is the identity.

Proof. Suppose for some $k, \alpha(k) \neq k$.

$$
\left\|T Z_{k}-Z_{k}\right\|_{p}=\left\|\Phi_{k}\left(z_{\alpha(k)}\right) T(1)-z_{k}\right\|_{p} \geq\left\|\Phi_{k}\left(z_{\alpha(k)}\right)-z_{k}\right\|_{p}-\|T(1)-1\|_{p}
$$

Thus

$$
\left\|T Z_{k}-Z_{k}\right\|_{p}>\left\|\Phi_{k}\left(z_{\alpha(k)}\right)-z_{k}\right\|_{p}-\left(\gamma_{p} / 2\right)
$$


However,

$$
\left\|\Phi_{k}\left(z_{\alpha(k)}\right)-z_{k}\right\|_{p}^{p}=\int_{K^{n}}\left|1-z_{k} \overline{\Phi_{k}\left(z_{\alpha(k)}\right)}\right|^{p} d m_{n}(z) .
$$

If we iterate the integral on the right, integrating first with respect to $d m_{1}\left(z_{k}\right)$, then, since $\alpha(k) \neq k$, we see that $\left\|\Phi_{k}\left(z_{\alpha(k)}\right)-z_{k}\right\|_{p}=\gamma_{p}$. This gives, in conjunction with (2.4), a contradiction.

We shall write $\alpha_{\mathrm{T}}$ to indicate the dependence on $T$ of the permutation $\alpha$ on the right of (1.4). We have the following result.

(2.5) Corollary. For $1 \leq p<\infty$, the sets $\left\{T \in G^{p}\left(U^{n}\right): \alpha_{T}=\mu\right\}$, where $\mu$ runs through all permutations of $\{1,2, \ldots, n\}$, are the distinct components of $G^{p}\left(U^{n}\right)$ with respect to the strong operator topology.

Proof. By (2.3) $\alpha_{T}$ must be constant on each component of $G^{p}\left(U^{n}\right)$. It is easy to see that for each permutation $\mu$ the corresponding set described above is arcwise connected in the strong operator topology.

Turning now to the uniform operator topology, we observe that elementary considerations show the following. For each positive integer $n$, there are positive constants $r_{n}$ and $R_{n}$ such that: if $0<\delta<1$ and $w_{0} \in S$, then

$$
r_{n} \delta^{2 n-1} \leq \sigma\left\{w \in S:\left|w-w_{0}\right|<\delta\right\} \leq R_{n} \delta^{2 n-1} .
$$

For arbitrary $w_{0} \in S$, let $f(z)=2^{-1}\left(1+\left\langle z, w_{0}\right\rangle\right)$ for $z \in \bar{B}_{n}$. Obviously $f\left(w_{0}\right)=1$, and the modulus of $f$ assumes its maximum only at $w_{0}$. By making use of the parallelogram identity, we find that for $0<\varepsilon<1$ :

$$
\begin{aligned}
\left\{w \in S:\left|w-w_{0}\right|<2 \varepsilon\right\} & \subseteq\{w \in S:|f(w)|>1-\varepsilon\} \\
& \subseteq\left\{w \in S:\left|w-w_{0}\right|<2 \sqrt{2} \sqrt{\varepsilon}\right\} .
\end{aligned}
$$

With these facts recorded for ease of reference in the next proof, we now state our next result.

(2.8) Theorem. Let $\psi \in H^{\infty}\left(B_{n}\right)$ and $\Phi \in \operatorname{Aut}\left(B_{n}\right)$. For $1 \leq p<\infty$, let $T$ be the bounded operator on $H^{p}\left(B_{n}\right)$ defined by $T f=\psi f(\Phi)$. If $\|T-I\|<1$, (where $I$ is the identity operator), then $\Phi(z)=z$ for all $z \in B_{n}$.

Proof. Suppose $\Phi$ is not the identity on $B_{n}$. Then for some $w_{0} \in S, \Phi\left(w_{0}\right) \neq w_{0}$. It follows from (2.7) that the sets $G_{k}=\left\{w \in S:|f(w)|>1-k^{-1}\right\},(k=1,2, \ldots)$, form a local base at $w_{0}$ in the topology of $S$. Since $\Phi\left(w_{0}\right) \neq w_{0}$, we can choose $j>1$ so that $\Phi\left(G_{j}\right)$ is disjoint from $G_{j}$. For $k \geq 3$, we see with the aid of (2.6) and (2.7) that $\left\|f^{k}\right\|_{p} \geq c k^{-(2 n-1) / p}$, where $c$ is a positive constant depending on $n$ and $p$. For notational convenience, write $V$ for the $G_{j}$ selected above. For $k \geq 3$, we have

$$
\|T-I\| \geq\left\{\int_{V}|f|^{k p} d \sigma\right\}^{1 / p} /\left\|f^{k}\right\|_{p}-\left\{\int_{V}\left|T\left(f^{k}\right)\right|^{p} d \sigma\right\}^{1 / p} /\left\|f^{k}\right\|_{p} .
$$


It is easy to see that $\left\{\int_{V}\left|T\left(f^{k}\right)\right|^{p} d \sigma\right\}^{1 / p} \leq\|\psi\|_{\infty}\left(1-j^{-1}\right)^{k}$. It follows from this and the above estimate for $\left\|f^{k}\right\|_{p}$ that the second term in the minorant of (2.9) approaches 0 as $k \rightarrow \infty$. Since $\left.\int_{V}\left|f^{k p} d \sigma=\left\|f^{k}\right\|_{p}^{p}-\int_{S \backslash V}\right| f\right|^{k p} d \sigma$, similar reasoning shows that the first term in the minorant of (2.9) tends to 1 . We get $\|T-I\| \geq 1$, which contradicts our hypothesis.

Remark. A more precise estimate of $\left\|f^{k}\right\|_{p}$ can be deduced if more sophisticated machinery than (2.6) and (2.7) is employed. Specifically (see [2]) for arbitrary $w_{0} \in S$, $k^{n-(1 / 2)}\left\|f^{k}\right\|_{p}^{p}$ approaches $(2 \pi)^{-1 / 2}(4 / p)^{n-(1 / 2)}[(n-1) !]$. Since the extra precision was not needed for the proof of $(2.8)$, we used more elementary estimates to make the paper more self-contained.

(2.10) Corollary. For $1 \leq p<\infty$, if $T$ is a composition operator on $H^{D}(U)$ and $\|T-I\|<1$, then $T=I$.

Proof. Since $\|T-I\|<1, T$ is invertible. It is known (see, e.g., [4]) that the composing function for an invertible composition operator belongs to $\operatorname{Aut}(U)$.

(2.11) Corollary. For $1 \leq p<\infty$ and $T \in G^{p}\left(B_{n}\right)$ the component of $T$ in $G^{p}\left(B_{n}\right)$, with respect to the uniform operator topology, is $\{\lambda T: \lambda \in K\}$.

Proof. By (2.8), $\left\{V \in G^{p}\left(B_{n}\right):\|V-I\|<1\right\}=\{\lambda I: \lambda \in K,|\lambda-1|<1\}$. It follows that $\{\lambda T: \lambda \in K\}$ is open in $G^{\mathrm{P}}\left(B_{n}\right)$. This set is obviously compact and connected.

(2.12) Theorem. For $1 \leq p<\infty$ and $T \in G^{p}\left(U^{n}\right)$, the component of $T$ in $G^{p}\left(U^{n}\right)$, with respect to the uniform operator topology, is $\{\beta T: \beta \in K\}$.

Proof. Suppose $V \in G^{\mathrm{p}}\left(U^{n}\right)$ and $\|V-I\|<\gamma_{\mathrm{p}} / 2$. By (2.3) $\alpha_{V}$ is the identity. Represent $V$ by (1.4). Suppose for some integer $\nu, 1 \leq \nu \leq n, \Phi_{\nu}$ is not the identity. Then we have a $w_{0} \in K$ such that $\Phi_{\nu}\left(w_{0}\right) \neq w_{0}$. Let $f(z)=2^{-1}\left(1+z \bar{w}_{0}\right)$ for $z \in \vec{U}$. As in the proof of Theorem (2.8) (for the special case $n=1$ ), $\left\|f^{k}\right\|_{p} \geq c k^{-1 / p}$ for $k \geq 3$, where $c$ is a positive constant. Also, for some $j>1,\left\{w \in K:|f(w)|>1-j^{-1}\right\}$ is disjoint from its $\Phi_{\nu}$-image. Denote this set by $J$. For $\left(z_{1}, \ldots, z_{n}\right) \in \mathbf{C}^{n}$, let $\hat{z}$ denote the $(n-1)$-tuple obtained by deleting $z_{v}$. Let $F(z)=f\left(z_{v}\right)$ for $z \in U^{n}$. For $k \geq 3$,

$$
\begin{aligned}
\|V-I\| \geq\left\{\int_{K^{n-1}} d m_{n-1}(\hat{z}) \int_{J} \mid F^{k p} d m_{1}\left(z_{\nu}\right)\right\}^{1 / p} /\left\|f^{k}\right\|_{p} & \\
& -\left\{\int_{K^{n-1}} d m_{n-1}(\hat{z}) \int_{J}\left|V\left(F^{k}\right)\right|^{p} d m_{1}\left(z_{\nu}\right)\right\}^{1 / p} /\left\|f^{k}\right\|_{p} .
\end{aligned}
$$

By direct calculation,

$$
\int_{J}\left|V\left(F^{k}\right)\right|^{p} d m_{1}\left(z_{\nu}\right) \leq\left[\prod_{i=1}^{n}\left\|\Phi_{i}^{\prime}\right\|_{\infty}\right] \int_{J}\left|f\left(\Phi_{\nu}\left(z_{\nu}\right)\right)\right|^{k p} d m_{1}\left(z_{\nu}\right) \leq\left[\prod_{i=1}^{n}\left\|\Phi_{i}^{\prime}\right\|_{\infty}\right]\left(1-j^{-1}\right)^{k p} .
$$

It follows that the second term in the minorant of (2.13) tends to 0 as $k \rightarrow \infty$. Similar considerations to those in the proof of (2.8) show that the first term in the minorant of (2.13) approaches 1 . So $1 \leq\|V-I\|<\gamma_{p} / 2$. But obviously from the definition $\gamma_{p} \leq 2$. This 
contradiction shows that $V=\lambda I$ for some $\lambda \in K$. We have established that

$$
\left\{V \in G^{p}\left(U^{n}\right):\|V-I\|<\gamma_{p} / 2\right\}=\left\{\lambda I: \lambda \in K, 4|\lambda-1|<\gamma_{p} / 2\right\}
$$

The proof can now be completed as was that of (2.11).

\section{Invariant Subspaces.}

(3.1) Theorem. For $1 \leq p<\infty 0$ and $H^{p}\left(B_{n}\right)$ (resp., $H^{p}\left(U^{n}\right)$ ) are the only closed invariant subspaces of $G^{p}\left(B_{n}\right)$ (resp., $G^{p}\left(U^{n}\right)$ ).

Proof. We take up $G^{p}\left(B_{n}\right)$ first. We shall write $d g$ for normalized Haar measure on the compact group $\Omega$ of all unitary operators on $\mathbf{C}^{n}$. For $f \in H^{p}\left(B_{n}\right)$ let $f^{\#}$ (sometimes called the radialization of $f$ ) be defined as the $H^{\mathrm{p}}\left(B_{n}\right)$-valued integral $\int_{\Omega} f \circ g^{-1} d g$, where "。" denotes composition. If $h \in \Omega$ and $z \in B_{n}, f^{\#}(h(z))=\int_{\Omega} f\left(\left(g^{-1} h\right)(z)\right) d g=f^{\#}(z)$. Since $f \in H^{\mathrm{P}}\left(B_{n}\right)$, it follows that $f^{\#}$ is a constant function. Hence $f^{\#}(z)=f(0)$ for all $z \in B_{n}$. Now suppose $M$ is a closed $G^{p}\left(B_{n}\right)$-invariant subspace. For each $g \in \Omega$, the operator on $H^{p}\left(B_{n}\right)$ given by composition with $g^{-1}$ obviously belongs to $G^{\mathrm{p}}\left(B_{n}\right)$. Hence if $f \in M$, then $f^{\#} \in M$. So either (i) $f(0)=0$ for all $f \in M$, or (ii) $M$ contains all constant functions. In case (i), if $F \in M$, and $F(a) \neq 0$ for some $a \in B_{n}$, then we can choose $\Phi \in \operatorname{Aut}\left(B_{n}\right)$ so that $\Phi(0)=a$. Then $\left\{\left(1-|a|^{2}\right) /(1-\langle z, a\rangle)^{2}\right\}^{n / p} F(\Phi(z))$ belongs to $M$. But this expression does not vanish for $z=0$. Hence in case (i). $M=0$. Now suppose case (ii) holds. For $0 \leq r<1$, let $\Phi_{r} \in \operatorname{Aut}\left(B_{n}\right)$ be given by $\Phi_{r}(z)=\left(1-r z_{1}\right)^{-1}\left(z_{1}-r, \sqrt{1-r^{2}} z_{2}, \ldots, \sqrt{1-r^{2}} z_{j}, \ldots\right.$, $\left.\sqrt{1-r^{2}} z_{n}\right)$. Let $T_{r} \in G^{\mathrm{P}}\left(B_{n}\right)$ be defined by (1.2) using $\Phi_{r}$ and taking $\lambda=1$. Let $S_{r}=$ $\left(1-r^{2}\right)^{-n / p} T_{r}$. Then $M$ is invariant under $S_{r}$, and $S_{r} f=\left(1-r z_{1}\right)^{-2 n / p} f\left(\Phi_{r}\right)$ for all $f \in H^{p}\left(B_{n}\right)$. If $P$ is any polynomial, then, as an $H^{p}\left(B_{n}\right)$-valued function of $r, S_{r}(P)$ has a derivative at $r=0$. Let $A(P)$ denote this derivative. For all $z \in B_{n}$,

$$
\{A(P)\}(z)=2 n p^{-1} z_{1} P(z)+P_{1}(z)\left(z_{1}^{2}-1\right)+\sum_{j=2}^{n} P_{i}(z) z_{1} z_{j}
$$

where $P_{i}$ denotes $\partial P / \partial z_{j}$. If $M$ contains a polynomial $P$, then $M$ also contains $A(P)$ by virtue of $S_{r}$-invariance. We shall prove by induction that for each integer $N \geq 0, M$ contains all monomials $\Pi_{j=1}^{n} z_{j}^{k_{i}}$ such that $\sum_{j=1}^{n} k_{j} \leq N$. This will show that $M=H^{\mathrm{p}}\left(B_{n}\right)$. The assertion is true for $N=0$ by the hypothesis for case (ii). Assume the assertion holds for $N$. If $\sum_{\mathrm{j}=1}^{n} k_{\mathrm{j}}$ does not exceed $N$, let $P$ be the corresponding monomial. $M$ contains $A(P)$. Using (3.2) we get that $z_{1} P \in M$. Using the invariance of $M$ under composition by unitaries we get that $z_{\mathrm{i}} P \in M$ for $j=1,2, \ldots, n$. The desired conclusion follows.

The assertion for $G^{\mathrm{p}}\left(U^{n}\right)$ can be established from the treatment in [1, §3] (which deals with $n=1$ ) by making obvious modifications. The crux of the analogy is to regard $L^{\mathrm{p}}\left(m_{n}\right)$ as a Banach algebra, with convolution as multiplication, and employ the Fejér kernel in $n$-variables (see, e.g., [5, XVII, 1]). 


\section{REFERENCES}

1. E. Berkson and H. Porta, The group of isometries of $H^{p}$, Ann. Mat. Pura Appl., (IV), CXIX, (1979), 231-238.

2. E. Berkson and H. Porta, The p-norms of peak functions, Proceedings of (Summer, 1978) Special Session on Operator Theory and Functional Analysis, Research Notes in Math., No. 38 (Pitman, 1979), 115-121.

3. A. Koranyi and S. Vagi, Isometries of $H^{p}$ spaces of bounded symmetric domains, Canad. $J$. Math., 28 (1976), 334-340.

4. H. Schwartz, Composition operators in $H^{p}$, Thesis, (University of Toledo, 1969).

5. A. Zygmund, Trigonometric Series (2nd edition), Vol. 2, (Cambridge University Press, 1959).

Department of Mathematics

UNIVERSTTY OF ILLINOIS

Urbana, ILlinOIS 61801 